\title{
Cognitive agency in sociocultural evolution
}

\section{A proposal to ground social modelling in the active inference framework}

\author{
Avel Guénin-Carlut \\ Kairos Research / Active Inference Lab
}




\begin{abstract}
Models of sociocultural evolution generally study the population dynamics of cultural traits given known biases in social learning. Cognitive agency, understood as the dynamics underlying a specific agent's adoption of a given trait, is essentially irrelevant in this framework. This article argues that although implementing and instrumenting agency in computational models is fundamentally challenging, it is ultimately possible and would help us overcome major limitations in our understanding of sociocultural dynamics.

Indeed, the behaviour of humans is not causally generated by a set of predefined behavioural laws, but by the situated activity of their cognitive architecture. Idealised models of biased transmission certainly help us understand specific features of population dynamics. However, they distract us from the deep entanglement of the cognitive and ecological processes underlying sociocultural evolution, and erase their embodied, subjective nature.

In line with the earlier “Thinking Through Other Minds" account of sociocultural evolution, this article highlights how the Active Inference framework can help us implement and instrument computational models that address these limitations. Such models would not only help ground our understanding of sociocultural evolution in the underlying cognitive dynamics, but also help solve (or frame) open questions in the study of ritual, relation between cultural transmission and innovation, as well as scales of cultural evolution.
\end{abstract}


"In a species capable of acquiring behavior by teaching or imitation, individuals are exposed to two different kinds of cues that they can use to solve local adaptive problems. Like any other organism, they can make inferences based on cues from the environment. However, they also observe the behaviors of a sample of their population. [...] Then, if the environment is not too variable, an adaptive psychology will evolve in which most people

ignore environmental cues and adopt behaviors that are common in the sample of the population they observe. They modify these behaviors rarely, or only at the margin, and as a result local adaptations evolve gradually often over many generations."

(Boyd et al., 2011, pp. 10922-10923)

"The main role of others in this kind of social learning is to direct attention rather than to convey specific semantic content (Tomasello 2014). In effect, social learning involves immersion in local contexts through what we call

regimes of attention and imitation that direct human agents to engage differentially in forms of shared intentionality. We have argued that such regimes of attention play a central role in the enculturation of human agents (Ramstead et al. 2016). Indeed, human beings seem particularly specialized for such forms of social learning (Sterelny 2012).”

(Veissière et al., 2020, sec. 2.3) 


\section{0 - Introduction}

With the democratisation of personal computing, the social sciences were able to develop a computational dimension (see eg Edelmann et al. (2020) for a broad overview). Those approaches broadly fall into two clusters: 1) analytical approaches, which recruit computational tools to build and analyse data (eg related to digital social networks) about the target system; 2) synthetic approaches, which attempt to build in silico experiments to display what behavior would predict a given model of the target system. Both of these meta-approaches can engage in a meaningful dialogue with existing social theory, but we will be interested in synthetic approaches specifically. This is because computational modelling helps cut unnecessary ambiguities in verbal theories (Healy, 2017; Smaldino, 2017) by forcing an explicit postulation of what structures are postulated by a specific scientific explanation. The phenomenon we observe in silico are entailed by the specific assumptions we have built into our model, and nothing more.

Therefore, computational modelling in the social sciences helps close the methodological gap with the so-called "natural" sciences. Biology and neurosciences indeed consider scientific explanation to constitute in the demonstration of the causal structures underlying a given phenomenon through the construction of explicit and context-specific models of the target system (Bechtel \& Abrahamsen, 2005; Craver, 2006; Mitchell, 2003). In key cases in biophysics where interaction structure is simple enough and stable through time, for example for the explanation of neuronal spiking through the Hodgkin-Huxley model, mathematical analysis or even back-of-theenvelope calculations can allow the solution of an explanatory model. This is is not the case for emergent, complex, highly fluctuating structures such as the formation of neuronal assemblies, which necessitates the use of either drastic simplificatory assumptions or principled computational modelling. Given that sociology is generally interested in structures of the last kind, computational modelling plays a necessary role in bridging the "natural" and social sciences.

However, an excessively acritical embrace of the computational turn of the social sciences presents specific dangers to the development of social theory. 
If we insist that proper scientific explanation must map onto specific computational models, then our scientific understanding of societies reduces to what we know how to model computationally. Even if no social scientist would adopt such an extreme reductive view, we should expect that the specific way we choose to translate scientific hypothesis about a target system into computational assumptions, those assumptions will make their way back into our understanding of the target system itself - even if they are acknowledged as simplificatory. It is widely acknowledged (although by no means universally (Craver, 2014)) that the role of models is not to accurately represent a system, but on the contrary to abstract away all its details except for the key structural properties that are relevant to our understanding (Smaldino, 2017; Wimsatt, 1987).

The core argument of this article is that social modellers have inadvertently abstracted away a core element of socio-cultural evolution, namely the cognitive agency of its actors. Indeed, socio-cultural evolution is generally modelled as either epidemiological or adaptive population dynamics, two closely related approaches which emphasize the explanatory role of the transmission process. When modellers dare to build explicit agents into their models, those agents are very simple automatons whose generative rules aim to capture documented cognitive biases, and consequently lack any identifiable relation with the subjective sense-making entailed by cognitive agency (Barandiaran et al., 2009; Moreno \& Mossio, 2015). This article attempts to show why this poses a key problem to our scientific understanding of sociocultural evolution, establish the Active Inference as an appropriate theoretical and computational framework to model the role of cognitive agency, and explain how it helps address open questions in the field of sociocultural evolution.

\section{1 - Social transmission without cognitive agency}

The computational modelling of socio-cultural evolution can be clustered into two broad categories: epidemiological and adaptive approaches. Both are closely related, in that they study the diffusion of cultural tokens given a certain set of transmission rules. Their main difference relies on the pragmatic role those tokens play in explaining behavior. Epidemiological 
approaches consider that tokens lack any adaptive role in shaping behavior, and accentuate the dynamics of diffusion on a given transmission network. Adaptive approaches, on the contrary, seek to explain the selection of pragmatically useful cultural tokens in the process of cultural diffusion. They typically do so by allowing cultural tokens to affect the cultural fitness of individuals, ie their capability to diffuse cultural tokens, and therefore to shape the topology of the social network entailed by social transmission. On the other hand, epidemiological approaches are essentially committed to downplay feedback from the properties of tokens to those of social networks.

This does not mean that epidemiological approaches must deny any role to the content of tokens. For example, Cultural Attraction Theory (CAT) is interested in how transmission biases shapes the content of cultural tokens by favoring the social diffusion of (and attraction toward) representations that are especially afferent to our evolved mind (Scott-Phillips et al., 2018; Sperber, 1994). For example, it is conjectured that such biases can explain the stability of bleeding as a medical practice or refusal to vaccinate belief despite their catastrophic counter-productivity in most cases (Miton et al., 2015; Miton \& Mercier, 2015). The content of tokens is however invoked here only as a phenomenon to be explained, not as a part of the explanation. By definition of epidemiological approaches, any causal role it plays is constrained to be irrelevant or epiphenomenal to the structural factors causing its emergence.

On the contrary, adaptive approaches consider the ecological intension of cultural tokens to play a central explanatory role in their diffusion. According to Gene-Culture Coevolution Theory (GCCT), contextual biases in social learning allow humans to discriminate where to find adaptive cultural tokens, most importantly toward accepted knowledge (Henrich \& Boyd, 1998), competent or prestigious individuals (Henrich \& Gil-White, 2001), as well as from committed displays of belief (Henrich, 2009). This set of cognitive biases enables cultural modes of evolutionary inheritance to interact constructively with genetic ones, and underly our ability to collectively and rapidly adapt to the wide range of environmental conditions typical of our species (Henrich, 2017; Henrich \& Muthukrishna, 2021). There again, even though cultural feedback on psychology is accepted and heavily 
accentuated (Heyes, 2012; Muthukrishna et al., 2021), cognitive biases are doing most of the explanatory work by enabling and constraining a process of runaway cultural adaptation.

The central role of cognitive biases in both CAT and GCCT is generally accepted as a given: surely collective socio-cultural dynamics must emerge from some kind of individual pattern of behavior. However, such a commitment is in no way trivial, and should elicit critical scrutiny. As such, a "cognitive bias" is only a deviation from normatively expected (ie rational, or neutral) behavior, that we know how to elicit and measure in constrained laboratory settings (Felin et al., 2019). They are not mechanical or generative models of cognitive activity like those developed by the biological or neurocognitive sciences, and the claim that they are anything more than phenomenons to be explained by calling onto underlying dynamics has dubious epistemological grounds (Bechtel \& Abrahamsen, 2005; Guénin--Carlut, 2020). However, they play a key role in abstracting away the active role of human agency in socio-cultural dynamics, and frame the problem in terms of social transmission and its constraints only.

The cases of CAT and GCCT stand out because of their strong Neo-Darwinian commitment. Both explicitly seek to explain socio-cultural dynamics in terms of the Modern Synthesis, which means they constrain themselves to call onto the population dynamics of socially transmitted traits. Their divergence in explanatory strategies essentially derives from a long-standing debate on whether cultural evolution should be understood as an adaptive process given its lack of individuated replicators (see Claidière et al. (2014) and Acerbi \& Mesoudi (2015)) '. However, both are centered on the way some measurable properties of social transmission entails some dynamical pattern in the diffusion of some cultural trait, as highlighted in Lewens (2015, Chapter 2). This entails a strong focus on the role of individuated behavioral regularities, highly idealised by construction and therefore prone to computational artefacts and circulation confirmation (Lewens, 2015, Chapter

1 we will leave aside any discussion on the nature of evolutionary information and its relation to adaptive dynamics, which would necessarily go far deeper in the technical details than the present article aims to. Let us point out however that it is generally agreed in evolutionary science that adaptative processes do not necessarily rely on the existence of individuated replicators (Jablonka \& Lamb, 2007; Laland et al., 2015). 
6), at the expense of the actual dynamics determining the agent's decisions or the subjective meaning it projects on its reality.

In contrast, computational sociology does not strongly commit to any ontological view. It tends to play fast and loose with the objects it builds into its models, drawing alternatively on highly idealised generative modelling and computational methods of statistical inference (Conte et al., 2012) to help formalise and test hypotheses drawn from mainstream sociology (Edelmann et al., 2020). There is consequently no principled reason to insist that computational sociology could not develop a research program around the role of agency in socio-cultural dynamics, and the prevalence of agent-based modelling in its methodology could even lead us to believe it already did so. This conclusion would however drastically misrepresent the meaning of the concept of agency, as the kind of agents that are typically implemented into computational models do not display anything like cognitive agency. Indeed, the process of agency in itself contradicts the role computational models typically play in our understanding of natural phenomena.

As claimed earlier, computational modelling enables the social sciences to build in silico experiments to test various hypotheses about the causes of documented phenomenons. As is standard in the experimental sciences, we use models to identify "natural laws", or in other terms measurable effects in the statistical relationship between some experimental observables. Sociocultural evolution is typically understood as affecting behavioral traits expressed in a human population, which we can reproduce in silico to measure their emergent effect. For example, perhaps cultural evolution can amplify small individual biases (Thompson et al., 2016), perhaps naive social learning (Henrich \& Boyd, 1998) and sparse patterns of socio-cultural transmission (Cantor et al., 2021; Migliano et al., 2020) facilitate cumulative cultural evolution, or perhaps between-group conflict drives the evolution of unequal or xenophobic human groups (Makowsky \& Smaldino, 2016). For the model to generate legible results, it needs to have clearly defined, easily measurable / manipulable parameters from which we can extract clear effects through statistical inference. 
The fundamental problem we would encounter while trying to implement agency in computational models of socio-cultural dynamics is that the very point of agency is to escape such legibility. Fundamentally, living systems are defined by a set of regularities (generally formalised as constraints or symmetries) which they create by their activity (Longo \& Montévil, 2013; Montévil \& Mossio, 2015). Agentive systems are living systems that make sense of their world in a way that continuously reconstructs the set of regularities they enact (Barandiaran et al., 2009; Moreno \& Mossio, 2015). This means that agents actively participate in defining the "behavioral laws" we can observe, and lack the kind of well defined, easily measurable / manipulable parameters we need to draw legible results from computational models or experimental approaches (Guénin--Carlut, 2020). Therefore, agency escapes by construction the core explanatory program of computational social sciences, and the study of its role in socio-cultural dynamics essentially remains terra incognita.

\section{2 - Implementing agency as unfolding cognitive landscapes}

The conclusion of the last section seems at the same time inescapable, and somewhat trivial. Of course, we cannot account for something as intangible as self-creation in every single model, but if we aim to explain any given sociocultural phenomenon we surely do not need to. The core goal of this section is to demonstrate that both counter-arguments are misguided: we can account for agency in computational modelling, and we need to. Indeed, a core goal of systems biology (and, incidentally, of sociology) is to find out how agents discover (or perhaps create) “adjacent possibles” (Kauffman, 2019) - literally states of matter that could come into being, but did not yet. This notion turns on its head our classical epistemology of natural laws and legible predictions, as the symmetries which are relevant to understand system evolution are not given a priori but rather generated by the system's endogenous activity (Longo et al., 2012).

This should not be understood as a principled statement of impenetrability, as it provides the basis for a positive (although historical) account of human 
sociocultural dynamics. Indeed, we know that our subjective perception of cultural traits shapes our willingness and ability to reproduce them, and is itself shaped by our earlier acculturation (Singh, 2020). We also know that our intentional engagement with our world through the process of Niche Construction (NC) is a key driver of our evolution (Laland \& O’Brien, 2011; Smith, 2013). After all, our ability to fire arrows and lay traps was not brought to us by the benevolent suggestion of some random mutation, but by the ecological activity of our ancestors, and now this ability feeds back onto every aspect of our lives. Understanding sociocultural dynamics does not therefore reduce to an elaborate account of social transmission, but also necessitates multiscale models of how we generate and engage with cultural traits (Singh et al., 2021).

Let us put aside the conceptual problems and turn back to formalism: what would be a minimal model for agency ? Let us begin by postulating a system with well-defined boundaries with its environment, effectively mediating all interactions. We can imagine something with eyes and claws, which can only interact by seeing what is in front of it and catching it. The stable existence of such a boundary entails that the internal and external states it defines will synchronise, so that each state of the boundary entails some expected state both in the system and its environment (Da Costa et al., 2021; K. Friston, 2019). Now, when some new state in the environment affects the boundary, it also affects the internal states of the system. Additionally, when some new state in the system affects its boundary, it also affects the environment. This process of integrating information and offloading it onto the environment is what we will call agency.

Critically, such agency is by essence an adaptive process, regardless of whether any kind of evolutionary inheritance is involved. Synchronisation across the boundary means that the agent-environment system will climb a free energy functional (Da Costa et al., 2021; K. Friston, 2019), corresponding to a natural fitness measure for the world model implicitly entailed by agent-environment dynamics (Ramstead et al., 2020)). For simpler systems, this reduces to the somewhat trivial claim that they will adopt their most stable form: water fits the shape of the recipients, stars follow their Newtonian course. Given that living systems' continued existence 
depends on how their engagement with the world enables them to continuously recreate their own structure, this means in their case that they will actively and intentionally attempt to stabilise their internal states by satisfying (implicit or explicit) expectations about what their sensori-motor flow should be (M. Kirchhoff et al., 2018; M. D. Kirchhoff \& Froese, 2017).

The neurocognitive approach committing to this formalism is generally known as the “Active Inference” (ActInf) framework. It essentially commits to predictive processing, ie the view that cognitive agents try to understand the world by building an internal model meant to "generate" the expected content of their sensorimotor flow, while grounding it in the basic dynamics of the agent-brain-world system (Bruineberg et al., 2018; M. Kirchhoff et al., 2018). It is most broadly known for its controversial ambition to integrate models of self-organisation, life, and cognition, for which it develops mathematical and computational models of how systems develop “representation" (Ramstead, Hesp, Tschantz, et al., 2021) (understood here as teleonomy in one's ecological and mental activity) through collective synchronisation (Palacios et al., 2017, 2019). It therefore affords a principled approach to integrating the large array of adaptive processes, including Darwinian selection, development, learning, niche construction, etc, in a single formal framework (Hesp et al., 2019; Ramstead et al., 2018, 2019; Ramstead, Hesp, Tschantz, et al., 2021).

Its most recognised achievements reside however in its historical neurocognitive bastion. Active Inference stands for an explicit mechanical model of human cognition (Badcock et al., 2019; K. Friston et al., 2016), which is understood to resolve theoretical and pragmatic questions regarding cognitive representations (Constant et al., 2021), action control (Hipólito, Baltieri, et al., 2021), agency (Clark, 2020), or phenomenology (Ramstead, Hesp, Sandved-Smith, et al., 2021). It does so by offering a multiscale model of how agents engage with their world (Bruineberg \& Rietveld, 2014), accounting for the entanglement of embodiment, ecological activity, enculturation, niche construction and phenomenology in the dynamics underlying cognition (Constant et al., 2019, 2020; Nave et al., 2020; Ramstead, Hesp, Sandved-Smith, et al., 2021). It therefore affords a natural model of collective cognition as the participative construction of cognitive 
landscapes which shape (and are shaped by) the engagement of agents, generating the alignment (or lack thereof) in cultural norms, behavior, etc.

This specific argument has recently been proposed, although at a narrative level, as a grounding account of cultural evolution (Veissière et al., 2020). The answers were broadly critical, which can be in part attributed to superficial confusion over the ecological-enactive (as opposed to representational) nature of the Active Inference framework (on this, see eg (Bruineberg et al., 2018; Constant et al., 2021; Ramstead et al., 2020)) and its account of historicity (see (Parr et al., 2021)). A deeper misalignment resides however in the assumption that Active Inference aims to replace CAT or GCCT as a competing explanation of sociocultural evolution. As such, Active Inference provides a computational and conceptual account of the human mind, as well as a formalism relating any population dynamics to a functional (adaptive) landscape they construct. It says nothing about how specific behavioral regularities can entail specific sociocultural regularity, and therefore does not do any explanatory work in and of itself.

What it does, however, is to provide us with the means to build computational models that help us understand the role of agency in sociocultural dynamics. Indeed, it provides a standard and explicit account of how to implement computational agents (Costa et al., 2020; Sajid et al., 2020), whose behavior derives from the activity of an explicit cognitvie architecture capable to generate a subjective understanding of its world and imprint this understanding back upon it. Importantly, the. The dynamics of a society of Active Inference agents therefore constitute an unfolding cognitive landscape, affording the modelling of open-ended sociocultural evolution, whose dynamics can be measured through a set of standard computational tools (K. J. Friston et al., 2020; Hipólito, Ramstead, et al., 2021; Palacios et al., 2019). We will hereby turn on the question of how to harness these features into concrete computational models that would help solve open questions in the study of sociocultural dynamics. 


\section{3 - Building agency-centered models of socio- cultural dynamics}

As earlier sections repeatedly pointed out, the question of how to implement, instrument, and interpret models displaying unfolding symmetries is very much an open one. In addition, no sociocultural phenomenon has a standard, consensual interpretation that could be unambiguously translated into a computational implementation. Most scientists interested in a given phenomenon do not quite agree on what they see in it, what the dynamics they postulate are like, and to build a model design which could clearly translate in everyone's language is the matter of hundreds of human-year of work. Attempting to single out here and now a model design that would most certainly provide a clear answer to theoretical debates on the proper theoretical understanding of such or such phenomenon is not only entirely beyond the scope of this article, it would most likely disregard important theoretical considerations and therefore impede future work on the matter.

What this section will attempt to do, however, is to provide proof of principle that the Active Inference framework would enable us to meaningfully translate some specific problems in the study of socio-cultural dynamics into legible in silico experiments. All those models could be defined as ActInf societies, as they will constitute in a bunch ActInf agents left to interact and make sense of their world together. Their implementation and instrumentation is straightforward when the said world is quite simple, as in opinion dynamics models (Albarracin et al., 2022). Most of the work we will do here is to define what kind of world could traduce some phenomenon of interest in our computational world. The following subsections will consequently discuss some open questions in sociocultural evolution, what kind of dynamics could be involved in the phenomenon of interest, and perhaps highlight basic elements of model design and instrumentation that would help answer them.

\section{1 - Embodied cognition in rituals and religion}

An important subfield of the cultural evolution literature studies religious belief and ritual activity, their cognitive underpinnings and their role in 
social organisation. While some insist that both are only a byproduct of earlier cognitive biases (Boyer, 2003; Boyer \& Liénard, 2006), others point to their adaptive role in collective organisation (Norenzayan et al., 2016; Sterelny, 2020). Ritual-like activity, like musical engagement and dancing, seems to constitute a core element of the human evolutionary complex through its role in emotional regulation and interpersonal synchronisation (Shilton et al., 2020). Religious ritual may canalise this phenomenon to enact a collective sense-making process grounding specific behaviors and norms within a social group, as field researchers generally insist. An explicit model of how embodied synchronisation, subjective phenomenology and explicit (normative or epistemic) belief may interact during a variety of ritual activities seems necessary to disentangle epidemiologic and adaptive, as well as ethnological and cognitive (Sterelny, 2018), interpretation of ritual engagement.

Strangely, evolutionary models of ritual and religion tend to avoid tackling this question, by presupposing the behavioral effect of ritual activity. For example, we know that answering collective dysphoric experience with stronger motivation to cooperation is adaptive (Whitehouse et al., 2017), and that orgiac rituals partially resetting social expectations could help find optimal solutions to intra-group conflicts (Froese et al., 2014; Froese \& Manzanilla, 2018), but not how these effects emerge from neurocognitive dynamics or subjective experience. An ActInf (or otherwise neurophenomenological) model of ritual engagement, accounting for all relevant scales from bodily dynamics to the cultural semantics of the ritual, would help us understand the ecological dynamics of ritual activity. It would even allow us to model directly the role of drugs such as serotoninergic or anticholinergic psychedelics (Fortier, 2018), marijuana, or the almost universal alcohol, in shaping ritual experience and incidentally social organisation.

\section{2 - Integrating evolutionary innovation and transmission}

As highlighted by modularist researchers, the process of adaptation through evolutionary selection entails both the ability to preferably transmit adaptive traits (inheritance) and the ability to generate new adaptive traits 
(evolvability) (A. Wagner, 2008; G. P. Wagner \& Altenberg, 1996). Classical evolutionary science traduces this dual process by giving a key explanatory role to the developmental process concretely generating those traits that come under selection (Bateson \& Laland, 2013). In contrast, cultural evolution literature generally discards entirely the question of how those variants are generated (with the notable exception of Charbonneau (2016) and the one computational model it inspired (Winters, 2019)), and chooses to focus instead on the condition under which adaptive variants can be selected in a population. The issue however resurfaces in interrogations on the relevance of an adaptive approach to cultural evolution given the fact that learner actually recreates (rather than copy) cultural traits (Claidière et al., 2014).

An explicit neurocognitive model of the development of "cultural traits" would go a long way to help us understand how such traits can be acquired or invented. A computational society of ActInf agents left to understand a common niche could each "innovate" by making up new generative models of their niche, but would also exchange representations by inferring each other's mental states through the imprint they leave on their niche (as underlined in Veissière et al. (2020)). A model of this kind would therefore provide a computational grounding for the dynamics underlying cultural innovation and reconstruction, while highlighting their deep intrication with (individual or collective) intentional behavior. It would also account for the bootstrapping of cultural evolution, accounting for a variety of narrative understandings based on shared intentionality (Tomasello \& Moll, 2010), theory of mind (Danchin et al., 2017) or bodily synchronisation (Shilton et al., 2020).

\section{3 - Distributed cognition and the scales of collective agency}

Humans are characterised by a high ecological reliance on collective dynamics, for example to infer adaptive cultural traits (Boyd et al., 2011; Henrich, 2017) or (as in the above ritual case) coordinated behavior (Shilton et al., 2020). Although human collectives, rather than individual humans, were pointed as the relevant scale to study some instances of cultural 
evolution (Muthukrishna \& Henrich, 2016; Smaldino, 2014; Smaldino \& Richerson, 2013), no formal approach exist at the moment to identify what scale is responsible for a given cognitive process. Such an approach could easily enough be imported from neurocomputational scaling methodology, from explicit quantification of emergence (Rosas et al., 2020) to renormalisation approaches of collective dynamics (K. J. Friston et al., 2020; Hipólito, Ramstead, et al., 2021). There are countless ways in which we could formalise collective agency or cognition, and measure what are the relevant scales of analysis for the emergence of a given process.

I have recently introduced a proposal based on the system biologic criterion for life and agency (Guénin--Carlut, 2021). In short, whatever scale of behavior has the capability to (re)produce its own structure should be understood as a locus for agency. Early City-States, with their self-produced urban infrastructure, rural backcountry, and centralised administration are a likely candidate for basic agency. This core idea can be instrumented through renormalisation approa ches drawn from the Active Inference formalism. We can identify the attractor to systems dynamics through classical field formalism, then reformulate the system's dynamics as this of its attractor, and reiterate until we identify a formulation meeting some criterion for agency (eg chaotic behavior K. J. Friston et al. (2020)). The scale of this formulation, properly speaking, drives the system's behavior. Importantly, field theory and renormalisation approaches constitute basic tools that can be refined endlessly to address scaling problems under any conceivable circumstances (in eco or in silico, top-down or bottom-up, etc).

\section{4 - Conclusion}

Agency plays a key role in sociocultural evolution. Cultural transmission is shaped by the subjective goal and understanding of their world developed by human agents, although Gene-Culture Coevolutionary Theory showed that their role in cumulative evolution could be productively reduced to wellknown cognitive biases. In contrast, cultural innovation is driven by intentional recombination of culturally laden and situated knowledge, and there is no sign yet that we could successfully model it with no reference to the headspace of our agents. Crucially, the human niche itself is constructed 
through the intentional and systematic environmental alteration to fit needs and expectations that are themselves the product of sociocultural evolution. In short, perhaps we can reduce the Darwinian struggle of individuated cultural variants to a handful of behavioral traits, but modelling the long run dynamics of culture entails a positive model of how human agents generate and engage with culturally laden cognitive landscapes.

At a conceptual level, nothing about it is complicated. The ecological and enactive approaches to cognitive sciences have long emphasized the role of embodied intelligence, subjective phenomenology and intentional engagement in human cognition. Even better, the recent predictive turn of neurocognitive has related these approaches with explicit mechanical models of how agents generate their understanding of reality. All we have to do is to replace sociocultural evolution in the context of its actual dynamical underpinnings, i.e. the cognitive activity of human societies, and investigate how multiple agents can mutually influence their generative models of and modes of engagement with the world. Crucially, such an approach provides us with a single framework to study the intrication of multiple scales in sociocultural evolution, from the embodied synchronisation characteristic of ritual activity to the collective sensemaking it entails, from the self-organisation of neuronal populations to the emergent behavior of City-State systems.

At a computational level, however, there is no reason for such optimism. Most of the time, we use computational models to document "natural laws" in the societies we build in silico, which we use to understand our world. For example, if people are naturally biased to reproduce cultural traits from prestigious sources, and in silico societies where people show such bias can consistently gradients in cultural fitness, then prestige bias can (at least in principle) explain the real-world phenomenon of cumulative culture. Agentive systems break this logic of legible effects and straightforward measure of results by developing and enacting a subjective understanding of the world, which traduces in irregular behavioral laws. Building models of the role of agency in sociocultural evolution entails a shift toward a different kind of social modelling, more intent on implementing complex computational societies whose organisation can thereafter be subject to exploratory analysis than on the end result of explaining specific effects. 
The present article proposes to implement computationally earlier conceptual research which recruited the Active Inference framework as a formal grounding for sociocultural evolution. One important advantage of this framework is that it integrates a computational model of agency synthesising insights from the predictive and enactive approaches to cognitive sciences with a mature toolset for dynamic causal inference, and more broadly exploratory analysis. In other words, it affords explicit modelling of agentive engagement with culturally laden cognitive landscapes, and of how concretely multiple scales of behavior participate in their evolution. The problem of open-ended evolution in societies of agents is ultimately an invariant of cognitive robotics, from evolutionary approaches (Doncieux et al., 2015) to swarm intelligence (Reina et al., 2021), which could perhaps be recruited instead of Active Inference for this project. What I argue is necessary is to explicit ground our understanding of sociocultural evolution in the bodily dynamics and intentional engagement underlying its dynamics.

\section{Acknowledgements}

This article was redacted in the context of the Active Inference Lab “Collective Intelligence” project. I would like to thank Natalie Kastel, Manon Job, Iona Brenac, Daniel Friedman, Jessica Balbuena, Lorena Sganzerla and Yseult Héjja-Brichard for their valuable comments. 


\section{Bibliography}

Acerbi, A., \& Mesoudi, A. (2015). If we are all cultural Darwinians what's the fuss about? Clarifying recent disagreements in the field of cultural evolution. Biology \& Philosophy, 30(4), 481-503. https://doi.org/10.1007/s10539-015-9490-2

Albarracin, M., Demekas, D., Ramstead, M., \& Heins, C. (2022). Epistemic Communities under Active Inference. https://doi.org/10.20944/preprints202201.0124.v1

Badcock, P. B., Friston, K. J., \& Ramstead, M. J. D. (2019). The hierarchically mechanistic mind: A free-energy formulation of the human psyche. Physics of Life Reviews, 31, 104-121. https://doi.org/10.1016/j.plrev.2018.10.002

Barandiaran, X. E., Di Paolo, E., \& Rohde, M. (2009). Defining Agency: Individuality, Normativity, Asymmetry, and Spatio-temporality in Action. Adaptive Behavior, 17(5), 367-386.

https://doi.org/10.1177/1059712309343819

Bateson, P., \& Laland, K. N. (2013). Tinbergen's four questions: An appreciation and an update. Trends in Ecology \& Evolution, 28(12), 712-718. https://doi.org/10.1016/j.tree.2013.09.013

Bechtel, W., \& Abrahamsen, A. (2005). Explanation: A mechanist alternative. Studies in History and Philosophy of Science Part C: Studies in History and Philosophy of Biological and Biomedical Sciences, 36(2), 421-441. https://doi.org/10.1016/j.shpsc.2005.03.010 
Boyd, R., Richerson, P. J., \& Henrich, J. (2011). The cultural niche: Why social learning is essential for human adaptation. Proceedings of the National Academy of Sciences, 108(Supplement 2), 10918-10925. https://doi.org/10.1073/pnas.1100290108

Boyer, P. (2003). Religious thought and behavior as by-products of brain function. Trends in Cognitive Sciences, 7(3), 119-124. https://doi.org/10.1016/S1364-6613(03)00031-7

Boyer, P., \& Liénard, P. (2006). Why ritualized behavior? Precaution Systems and action parsing in developmental, pathological and cultural rituals. Behavioral and Brain Sciences, 29(6), 595-613. https://doi.org/10.1017/S0140525X06009332

Bruineberg, J., Kiverstein, J., \& Rietveld, E. (2018). The anticipating brain is not a scientist: The free-energy principle from an ecological-enactive perspective. Synthese, 195(6), 2417-2444. https://doi.org/10.1007/s11229-016-1239-1

Bruineberg, J., \& Rietveld, E. (2014). Self-organization, free energy minimization, and optimal grip on a field of affordances. Frontiers in Human Neuroscience, 8. https://doi.org/10.3389/fnhum.2014.00599

Cantor, M., Chimento, M., Smeele, S. Q., He, P., Papageorgiou, D., Aplin, L. M., \& Farine, D. R. (2021). Social network architecture and the tempo of cumulative cultural evolution. Proceedings of the Royal Society B: Biological Sciences, 288(1946), 20203107. https://doi.org/10.1098/rspb.2020.3107 
Charbonneau, M. (2016). Modularity and Recombination in Technological Evolution. Philosophy \& Technology, 29(4), 373-392. https://doi.org/10.1007/s13347-016-0228-0

Claidière, N., Scott-Phillips, T. C., \& Sperber, D. (2014). How Darwinian is cultural evolution? Philosophical Transactions of the Royal Society B: Biological Sciences, 369(1642), 20130368. https://doi.org/10.1098/rstb.2013.0368

Clark, A. (2020). Beyond Desire? Agency, Choice, and the Predictive Mind. Australasian Journal of Philosophy, 98(1), 1-15. https://doi.org/10.1080/00048402.2019.1602661

Constant, A., Clark, A., \& Friston, K. J. (2021). Representation Wars: Enacting an Armistice Through Active Inference. Frontiers in Psychology, 11, 3798. https://doi.org/10.3389/fpsyg.2020.598733

Constant, A., Clark, A., Kirchhoff, M., \& Friston, K. J. (2019). Extended active inference: Constructing predictive cognition beyond skulls. Mind \& Language, n/a(n/a). https://doi.org/10.1111/mila.12330

Constant, A., Tschantz, A., Millidge, B., Criado-Boado, F., Martinez, L. M., Müller, J., \& Clark, A. (2020). The Acquisition of Culturally Patterned Attention Styles under Active Inference. PsyArXiv. https://doi.org/10.31234/osf.io/rchaf

Conte, R., Gilbert, N., Bonelli, G., Cioffi-Revilla, C., Deffuant, G., Kertesz, J., Loreto, V., Moat, S., Nadal, J.-P., Sanchez, A., Nowak, A., Flache, A., San Miguel, M., \& Helbing, D. (2012). Manifesto of 
computational social science. The European Physical Journal Special Topics, 214(1), 325-346. https://doi.org/10.1140/epjst/e2012-01697-8

Costa, L. D., Parr, T., Sajid, N., Veselic, S., Neacsu, V., \& Friston, K. J. (2020). Active inference on discrete state-spaces: A synthesis. Journal of Mathematical Psychology. https://doi.org/10.1016/j.jmp.2020.102447

Craver, C. F. (2006). When mechanistic models explain. Synthese, 153(3), 355-376. https://doi.org/10.1007/s11229-006-9097-x

Craver, C. F. (2014). The ontic conception of scientific explanation. Explanation in the Biological and Historical Sciences. Dordrecht, Netherlands: Springer, 27-52.

Da Costa, L., Friston, K., Heins, C., \& Pavliotis, G. A. (2021). Bayesian Mechanics for Stationary Processes. ArXiv:2106.13830 [Math-Ph, Physics:Nlin, q-Bio]. http://arxiv.org/abs/2106.13830

Danchin, É., Giraldeau, L.-A., Valone, T. J., \& Wagner, R. R. (2017). Public Information: From Nosy Neighbors to Cultural Evolution.

Doncieux, S., Bredeche, N., Mouret, J.-B., \& Eiben, A. E. (Gusz). (2015). Evolutionary Robotics: What, Why, and Where to. Frontiers in Robotics and AI, 2. https://doi.org/10.3389/frobt.2015.00004

Edelmann, A., Wolff, T., Montagne, D., \& Bail, C. A. (2020). Computational Social Science and Sociology. Annual Review of Sociology, 46(1), 6181. https://doi.org/10.1146/annurev-soc-121919-054621

Felin, T., Felin, M., Krueger, J. I., \& Koenderink, J. (2019). On SurpriseHacking. Perception, 48(2), 109-114. https://doi.org/10.1177/0301006618822217 
Fortier, M. (2018). Le façonnement neuropharmacologique de la culture. Anthropologie comparée des rituels à hallucinogènes sérotoninergiques et anticholinergiques. Cahiers d'anthropologie sociale, $N^{\circ} 17(2), 132-$ 151.

Friston, K. (2019). A free energy principle for a particular physics.

ArXiv:1906.10184 [q-Bio]. http://arxiv.org/abs/1906.10184

Friston, K., FitzGerald, T., Rigoli, F., Schwartenbeck, P., \& Pezzulo, G. (2016). Active Inference: A Process Theory. Neural Computation, 29(1), 1-49. https://doi.org/10.1162/NECO_a_00912

Friston, K. J., Fagerholm, E. D., Zarghami, T. S., Parr, T., Hipólito, I., Magrou, L., \& Razi, A. (2020). Parcels and particles: Markov blankets in the brain. ArXiv:2007.09704 [q-Bio].

http://arxiv.org/abs/2007.09704

Froese, T., Gershenson, C., \& Manzanilla, L. R. (2014). Can Government Be Self-Organized? A Mathematical Model of the Collective Social Organization of Ancient Teotihuacan, Central Mexico. PLOS ONE, 9(10), e109966. https://doi.org/10.1371/journal.pone.0109966

Froese, T., \& Manzanilla, L. R. (2018). Modeling collective rule at ancient Teotihuacan as a complex adaptive system: Communal ritual makes social hierarchy more effective. Cognitive Systems Research, 52, 862874. https://doi.org/10.1016/j.cogsys.2018.09.018

Guénin--Carlut, A. (2020). Cognition in eco \& cognition in vitro: Can cognitive science explain ecological behavior? https://doi.org/10.17605/OSF.IO/ERCZ6 
Guénin--Carlut, A. (2021). Thinking like a State-Embodied intelligence in the deep history of our collective minds. OSF Preprints. https://doi.org/10.31219/osf.io/dxnzt

Healy, K. (2017). Fuck Nuance. Sociological Theory, 35(2), 118-127. https://doi.org/10.1177/0735275117709046

Henrich, J. (2009). The evolution of costly displays, cooperation and religion: Credibility enhancing displays and their implications for cultural evolution. Evolution and Human Behavior, 30(4), 244-260. https://doi.org/10.1016/j.evolhumbehav.2009.03.005

Henrich, J. (2017). The Secret of Our Success: How Culture Is Driving Human Evolution, Domesticating Our Species, and Making Us Smarter. Princeton University Press.

Henrich, J., \& Boyd, R. (1998). The Evolution of Conformist Transmission and the Emergence of Between-Group Differences. Evolution and Human Behavior, 19(4), 215-241. https://doi.org/10.1016/S10905138(98)00018-X

Henrich, J., \& Gil-White, F. J. (2001). The evolution of prestige: Freely conferred deference as a mechanism for enhancing the benefits of cultural transmission. Evolution and Human Behavior, 22(3), 165-196. https://doi.org/10.1016/S1090-5138(00)00071-4

Henrich, J., \& Muthukrishna, M. (2021). The Origins and Psychology of Human Cooperation. Annual Review of Psychology, 72(1), 207-240. https://doi.org/10.1146/annurev-psych-081920-042106 
Hesp, C., Ramstead, M., Constant, A., Badcock, P., Kirchhoff, M., \& Friston, K. (2019). A Multi-scale View of the Emergent Complexity of Life: A Free-Energy Proposal. In G. Y. Georgiev, J. M. Smart, C. L. Flores Martinez, \& M. E. Price (Eds.), Evolution, Development and Complexity (pp. 195-227). Springer International Publishing. https://doi.org/10.1007/978-3-030-00075-2_7

Heyes, C. (2012). Grist and mills: On the cultural origins of cultural learning. Philosophical Transactions of the Royal Society B: Biological Sciences, 367(1599), 2181-2191. https://doi.org/10.1098/rstb.2012.0120

Hipólito, I., Baltieri, M., Friston, K., \& Ramstead, M. J. D. (2021). Embodied skillful performance: Where the action is. Synthese. https://doi.org/10.1007/s11229-020-02986-5

Hipólito, I., Ramstead, M. J. D., Convertino, L., Bhat, A., Friston, K., \& Parr, T. (2021). Markov blankets in the brain. Neuroscience \& Biobehavioral Reviews. https://doi.org/10.1016/j.neubiorev.2021.02.003 Jablonka, E., \& Lamb, M. J. (2007). Précis of Evolution in Four Dimensions. Behavioral and Brain Sciences, 30(4), 353-365. https://doi.org/10.1017/S0140525X07002221

Kauffman, S. A. (2019). A World Beyond Physics: The Emergence and Evolution of Life. Oxford University Press.

Kirchhoff, M. D., \& Froese, T. (2017). Where There is Life There is Mind: In Support of a Strong Life-Mind Continuity Thesis. Entropy, 19(4), 169. https://doi.org/10.3390/e19040169 
Kirchhoff, M., Parr, T., Palacios, E., Friston, K., \& Kiverstein, J. (2018). The Markov blankets of life: Autonomy, active inference and the free energy principle. Journal of The Royal Society Interface, 15(138), 20170792. https://doi.org/10.1098/rsif.2017.0792

Laland, K. N., \& O’Brien, M. J. (2011). Cultural Niche Construction: An Introduction. Biological Theory, 6(3), 191-202. https://doi.org/10.1007/s13752-012-0026-6

Laland, K. N., Uller, T., Feldman, M. W., Sterelny, K., Müller, G. B., Moczek, A., Jablonka, E., \& Odling-Smee, J. (2015). The extended evolutionary synthesis: Its structure, assumptions and predictions. Proc. R. Soc. B, 282(1813), 20151019. https://doi.org/10.1098/rspb.2015.1019

Lewens, T. (2015). Cultural Evolution: Conceptual Challenges. OUP Oxford. Longo, G., \& Montévil, M. (2013). Extended criticality, phase spaces and enablement in biology. Chaos, Solitons \& Fractals, 55, 64-79. https://doi.org/10.1016/j.chaos.2013.03.008

Longo, G., Montévil, M., \& Kauffman, S. (2012). No entailing laws, but enablement in the evolution of the biosphere. Proceedings of the 14th Annual Conference Companion on Genetic and Evolutionary Computation, 1379-1392. https://doi.org/10.1145/2330784.2330946

Makowsky, M. D., \& Smaldino, P. E. (2016). The evolution of power and the divergence of cooperative norms. Journal of Economic Behavior \& Organization, 126, 75-88. https://doi.org/10.1016/j.jebo.2015.09.002 
Migliano, A. B., Battiston, F., Viguier, S., Page, A. E., Dyble, M., Schlaepfer, R., Smith, D., Astete, L., Ngales, M., Gomez-Gardenes, J., Latora, V., \& Vinicius, L. (2020). Hunter-gatherer multilevel sociality accelerates cumulative cultural evolution. Science Advances, 6(9), eaax5913. https://doi.org/10.1126/sciadv.aax5913

Mitchell, S. D. (2003). Biological Complexity and Integrative Pluralism. Cambridge University Press.

Miton, H., Claidière, N., \& Mercier, H. (2015). Universal cognitive mechanisms explain the cultural success of bloodletting.

Miton, H., \& Mercier, H. (2015). Cognitive Obstacles to Pro-Vaccination Beliefs.

Montévil, M., \& Mossio, M. (2015). Biological organisation as closure of constraints. Journal of Theoretical Biology, 372, 179-191. https://doi.org/10.1016/j.jtbi.2015.02.029

Moreno, A., \& Mossio, M. (2015). Agency. In A. Moreno \& M. Mossio (Eds.), Biological Autonomy: A Philosophical and Theoretical Enquiry (pp. 89-109). Springer Netherlands. https://doi.org/10.1007/978-94-017$9837-2 \_4$

Muthukrishna, M., \& Henrich, J. (2016). Innovation in the collective brain. Philosophical Transactions of the Royal Society B: Biological Sciences, 371(1690), 20150192. https://doi.org/10.1098/rstb.2015.0192

Muthukrishna, M., Henrich, J., \& Slingerland, E. (2021). Psychology as a Historical Science. Annual Review of Psychology, 72(1), 717-749. https://doi.org/10.1146/annurev-psych-082820-111436 
Nave, K., Deane, G., Miller, M., \& Clark, A. (2020). Wilding the predictive brain. WIREs Cognitive Science, 11(6), e1542. https://doi.org/10.1002/wcs.1542

Norenzayan, A., Shariff, A. F., Gervais, W. M., Willard, A. K., McNamara, R. A., Slingerland, E., \& Henrich, J. (2016). The cultural evolution of prosocial religions. Behavioral and Brain Sciences, 39. https://doi.org/10.1017/S0140525X14001356

Palacios, E. R., Isomura, T., Parr, T., \& Friston, K. (2019). The emergence of synchrony in networks of mutually inferring neurons. Scientific Reports, 9(1), 6412. https://doi.org/10.1038/s41598-019-42821-7

Palacios, E. R., Razi, A., Parr, T., Kirchhoff, M., \& Friston, K. (2017). Biological Self-organisation and Markov blankets. BioRxiv, 227181. https://doi.org/10.1101/227181

Parr, T., Da Costa, L., Heins, C., Ramstead, M. J. D., \& Friston, K. J. (2021). Memory and Markov Blankets. Entropy, 23(9), 1105. https://doi.org/10.3390/e23091105

Ramstead, M. J. D., Badcock, P. B., \& Friston, K. J. (2018). Answering Schrödinger's question: A free-energy formulation. Physics of Life Reviews, 24, 1-16. https://doi.org/10.1016/j.plrev.2017.09.001

Ramstead, M. J. D., Constant, A., Badcock, P. B., \& Friston, K. J. (2019). Variational ecology and the physics of sentient systems. Physics of Life Reviews, 31, 188-205. https://doi.org/10.1016/j.plrev.2018.12.002 Ramstead, M. J. D., Hesp, C., Sandved-Smith, L., Mago, J., Lifshitz, M., Pagnoni, G., Smith, R., Dumas, G., Lutz, A., Friston, K., \& Constant, 
A. (2021). From generative models to generative passages: $A$ computational approach to (neuro)phenomenology. PsyArXiv. https://doi.org/10.31234/osf.io/k9pbn

Ramstead, M. J. D., Hesp, C., Tschantz, A., Smith, R., Constant, A., \& Friston, K. J. (2021). Neural and phenotypic representation under the free-energy principle. Neuroscience \& Biobehavioral Reviews. https://doi.org/10.1016/j.neubiorev.2020.11.024

Ramstead, M. J. D., Kirchhoff, M. D., \& Friston, K. J. (2020). A tale of two densities: Active inference is enactive inference. Adaptive Behavior, 28(4), 225-239. https://doi.org/10.1177/1059712319862774

Reina, A., Ferrante, E., \& Valentini, G. (2021). Collective decision-making in living and artificial systems: Editorial. Swarm Intelligence, 15(1), 1-6. https://doi.org/10.1007/s11721-021-00195-5

Rosas, F. E., Mediano, P. A. M., Jensen, H. J., Seth, A. K., Barrett, A. B., Carhart-Harris, R. L., \& Bor, D. (2020). Reconciling emergences: An information-theoretic approach to identify causal emergence in multivariate data. PLOS Computational Biology, 16(12), e1008289. https://doi.org/10.1371/journal.pcbi.1008289

Sajid, N., Ball, P. J., Parr, T., \& Friston, K. J. (2020). Active inference: Demystified and compared. ArXiv:1909.10863 [Cs, q-Bio]. http://arxiv.org/abs/1909.10863

Scott-Phillips, T., Blancke, S., \& Heintz, C. (2018). Four misunderstandings about cultural attraction. Evolutionary Anthropology: Issues, News, and Reviews, 27(4), 162-173. https://doi.org/10.1002/evan.21716 
Shilton, D., Breski, M., Dor, D., \& Jablonka, E. (2020). Human Social Evolution: Self-Domestication or Self-Control? Frontiers in Psychology, 11. https://doi.org/10.3389/fpsyg.2020.00134

Singh, M. (2020). Subjective selection and the evolution of complex culture. PsyArXiv. https://doi.org/10.31234/osf.io/4t2ud

Singh, M., Acerbi, A., Caldwell, C. A., Danchin, É., Isabel, G., Molleman, L., Scott-Phillips, T., Tamariz, M., van den Berg, P., van Leeuwen, E. J. C., \& Derex, M. (2021). Beyond social learning. Philosophical Transactions of the Royal Society B: Biological Sciences, 376(1828), rstb.2020.0050, 20200050. https://doi.org/10.1098/rstb.2020.0050

Smaldino, P. E. (2014). The cultural evolution of emergent group-level traits. Behavioral and Brain Sciences, 37(3), 243-254. https://doi.org/10.1017/S0140525X13001544

Smaldino, P. E. (2017). Models Are Stupid, and We Need More of Them. In Computational Social Psychology. Routledge.

Smaldino, P. E., \& Richerson, P. J. (2013). Human Cumulative Cultural Evolution as a Form of Distributed Computation. In P. Michelucci (Ed.), Handbook of Human Computation (pp. 979-992). Springer. https://doi.org/10.1007/978-1-4614-8806-4_76

Smith, E. A. (2013). Agency and Adaptation: New Directions in Evolutionary Anthropology. Annual Review of Anthropology, 42(1), 103-120. https://doi.org/10.1146/annurev-anthro-092412-155447

Sperber, D. (1994). The epidemiology of representations. Mapping the Mind: Domain Specificity in Cognition and Culture, 39. 
Sterelny, K. (2018). Religion re-explained. Religion, Brain \& Behavior, 8(4), 406-425. https://doi.org/10.1080/2153599X.2017.1323779

Sterelny, K. (2020). Religion: Costs, signals, and the Neolithic transition. Religion, Brain \& Behavior, 10(3), 303-320. https://doi.org/10.1080/2153599X.2019.1678513

Thompson, B., Kirby, S., \& Smith, K. (2016). Culture shapes the evolution of cognition. Proceedings of the National Academy of Sciences, 113(16), 4530-4535. https://doi.org/10.1073/pnas.1523631113

Tomasello, M., \& Moll, H. (2010). The Gap is Social: Human Shared Intentionality and Culture. In P. M. Kappeler \& J. Silk (Eds.), Mind the Gap: Tracing the Origins of Human Universals (pp. 331-349). Springer. https://doi.org/10.1007/978-3-642-02725-3_16

Veissière, S. P. L., Constant, A., Ramstead, M. J. D., Friston, K. J., \& Kirmayer, L. J. (2020). Thinking through other minds: A variational approach to cognition and culture. Behavioral and Brain Sciences, 43. https://doi.org/10.1017/S0140525X19001213

Wagner, A. (2008). Robustness and evolvability: A paradox resolved. Proceedings of the Royal Society B: Biological Sciences, 275(1630), 91-100. https://doi.org/10.1098/rspb.2007.1137

Wagner, G. P., \& Altenberg, L. (1996). Perspective: Complex Adaptations and the Evolution of Evolvability. Evolution, 50(3), 967-976. https://doi.org/10.1111/j.1558-5646.1996.tb02339.x

Whitehouse, H., Jong, J., Buhrmester, M. D., Gómez, Á., Bastian, B., Kavanagh, C. M., Newson, M., Matthews, M., Lanman, J. A., McKay, 
R., \& Gavrilets, S. (2017). The evolution of extreme cooperation via shared dysphoric experiences. Scientific Reports, 7, 44292. https://doi.org/10.1038/srep44292

Wimsatt, W. C. (1987). False models as means to truer theories. Neutral Models in Biology, 23-55.

Winters, J. (2019). Escaping optimization traps: The role of cultural adaptation and cultural exaptation in facilitating open-ended cumulative dynamics. Palgrave Communications.

https://doi.org/10.1057/s41599-019-0361-3 